\title{
D-STRUCTURES AND DERIVED KOSZUL DUALITY FOR UNITAL OPERAD ALGEBRAS
}

\author{
TYLER FOSTER, PO HU AND IGOR KRIZ
}

\begin{abstract}
Generalizing a concept of Lipshitz, Ozsváth and Thurston from Bordered Floer homology, we define $D$-structures on algebras of unital operads, which can also be interpreted as a generalization of a seemingly unrelated concept of Getzler and Jones. This construction gives rise to an equivalence of derived categories, which can be thought of as a unital version of Koszul duality using non-unital Quillen homology. We also discuss a multi-sorted version of the construction, which provides a framework for unifying the known algebraic contexts of Koszul duality.
\end{abstract}

\section{INTRODUCTION}

Koszul duality for operads and algebras over them was introduced in the landmark paper [10] by Ginzburg and Kapranov. For example, as long as we work over a field of characteristic 0, the operads encoding commutative and Lie algebras are Koszul dual, while the operad encoding associative algebras is self-dual. The Koszul duality of Ginzburg and Kapranov [10] has both a non-derived and a derived version. The derived version involves a kind of a bar construction on an operad, which we call the Ginzburg-Kapranov bar construction. The Ginzburg-Kapranov bar construction on an operad is a differential graded co-operad, which, under suitable finiteness assumptions, is the dual of a differential graded operad, the derived Koszul dual of the original operad. When there is not enough finiteness to dualize, the DG co-operad can also be used directly, and we refer to it as the Koszul transform of the original operad. Just as in the more classical Koszul duality of associative algebras (cf. [23]), there is a property of an operad being Koszul which implies that the Koszul dual operad is, in fact, quasi-isomorphic to a (non-differential graded) operad, which is then called the non-derived Koszul dual.

The concept of derived Koszul duality is (as always) more important to homotopy theory foundations than the corresponding non-derived concept, although the non-derived concept is a useful calculational tool. Derived Koszul dual operads are a part of a more general scheme, which 
in some sense goes back to Quillen [24]: In any based category $C$ with finite products, we may define the category of abelian objects $A b(C)$. In good (and also typical) cases, the forgetful functor

$$
U: A b(C) \rightarrow C
$$

has a left adjoint $L$, called abelianization. The functor $L U$ is then a co-monad in $A b(C)$. In the presence of a mechanism creating derived categories, if we denote by $M$ the derived version of the co-monad $L U$, then the Koszul transform of the derived category $D C$ should be the category of $M$-co-algebras. This is, of course, somewhat vague, and it gets even more so: Abelian objects may be replaced by $E_{\infty}$-objects, and ultimately, the functor $L$ may be replaced by Dwyer-Kan stabilization (an anague of the topological notion of spectra in a based category with certain additional structure, cf. [3]). It is very difficult to get any precise theorems along these lines in the most general case because of convergence problems in the associated cobar spectral sequence. Nevertheless, it was observed by Kontsevich that derived Koszul duality for operads is a part of this general scenario (see [14, 15, 13, 12]).

One of the puzzles of Koszul duality has been that it does not seem to interact well with units. The main problem is that unital (nonaugmented) operads, algebras over such operads etc. do not tend to form a based category, and therefore the scheme described in the last paragraph does not work directly. (When discussing a unital context, we mean, similarly as [24], that there is a unit but not an augmentation. Similarly as for algebras, the category of augmented unital operads is canonically equivalent to the category of non-unital operads by passing to the augmentation ideal.) Furthermore, if one takes Quillen cohomology of unital objects in the corresponding non-unital category, one usually gets zero ([24]). It has been an open question whether there is a version of Koszul duality which works, say, for unital algebras over unital operads, in a sense which would generalize the above categorical scheme.

The main result of the present paper is to define derived Koszul duality for unital algebras over unital operads. Our approach is to take non-unital homology of the unital $\mathcal{C}$-algebra, which by [14, 15] is calculated by the Ginzburg-Kapranov bar construction. Even though these bar constructions have 0 chain homology, they have however additional structure (which we call D-structure). There is a natural way of defining morphisms and equivalences of $D$-structures, and prove that the resulting derived category is equivalent to the category of $\mathcal{C}$-algebras. Comment: while we do rigorously construct the derived category of $D$-structures for a general unital operad, we do not construct a Quillen 
model structure [4] for them. Constructing such a structure remains an interesting open problem.

The term D-structure is taken from the bordered Floer homology of Lipshitz, Oszváth and Thurston [17], which is a (pointed) topological quantum field theory built out of the Heegaard-Floer homology invented by Ozsváth and Szabó [21]. From the point of view of the present paper, they use our derived Koszul duality in the special case of $A$-modules where $A$ is a unital algebra: both the modules and their Koszul transform $D$-structures occur naturally as combinatorial objects calculating Bordered Floer homology. There are a few technical nuances, for example, on one side, [17] consider $A_{\infty}$-modules instead of strict modules, and also consider the differential as one of the operations. We replace this by a context most suitable to our techniques; the different variants of the concepts, both on the level of modules and $D$-structure, are easily seen to lead to equivalent derived categories. In any case, it is very important to [17] to have an equivalence of derived categories; they need to construct a pairing of two objects in a certain geometrically given category, while a natural geometrically given pairing is between an object of the category and an object of its Koszul transform. We reproduce a full generalization of this equivalence of categories to the general context of unital algebras over unital operads.

Interestingly enough, $D$-structures turn out to be also equivalent to the concept of quasi-free (or almost free) operad algebras introduced, in the augmented case, in 1994 by Getzler and Jones in their unpublished preprint [9] (see also Fresse [6]). We only need to notice that the concept makes sense in the non-augmented case. Of course, it is the more explicit $D$-structure version of the concept which occurs naturally in [17, and is naturally interpreted as a version of Koszul duality. The equivalence of both concepts is an easy but very pretty observation, a clean and simple pattern in what has become known as a somewhat messy and technical area.

Since many papers were written on this subject to date, and the basic background definitions are not unified (or even always completely rigorous), we chose to make the present paper self-contained, and to deal with a number of technical issues. Ginzburg and Kapranov [10] work over a field of characteristic 0, while Lipshitz, Ozsváth and Thurston [17] work over a field of characteristic 2. The motivation of [17] is to avoid the discussion of signs. In this paper, we do work out the signs, and in a sense, this is one of our key points: While in [10], a clever exterior algebra method is introduced to control the signs in the Ginzburg-Kapranov bar construction, we need to extend this construction by a Clifford algebra to handle the differential graded case. 
This becomes important because the derived Koszul transform of a DG operad is, in fact, naturally bigraded. We need to totalize this into a singly graded chain complex. While there are standard signs for totalizing double chain complexes, these signs do not work for generalizing the D-structures of Lipshitz, Ozsváth and Thurston [17] outside of characteristic 2! The Clifford algebra method we use reconciles the signs of the internal grading with the Ginzburg-Kapranov bar construction completely, and works in the setting we need.

To make a rigorous treatment of the signs, we introduce our own rigorous definition of "planar trees". Many previous papers rely only on pictorial intuition, which, in some cases, even obscures the crucial point of the role of identifications introduced by symmetric group actions on operads. This is related to the more general point that while we are interested in derived categories, $D$-structure is a point-set level algebraic structure, and therefore for our purpose, objects cannot be freely replaced by quasiisomorphic ones (this is even more apparent when we use the interpretation of [9]). This is one of the reasons why we must use the Ginzburg-Kapranov bar construction [10]; we do not know how to make our theory replacing it with a two-sided bar construction of monads [19], (which, of course, could be used if we worked only up to homotopy). In some sense, [10] deal with the same issue in their original concept of non-unital Koszul duality.

A related point is that the reason Ginzburg-Kapranov [10] work in characteristic 0 is that otherwise the monad associated with an operad does not preserve quasiisomorphisms. This happens in some very basic cases, for example for commutative algebras. This is a very well recognized phenomenon which plays an important role in homotopy theory (cf. [20], or, in an algebraic context, [16]). In this paper, we work in the category of modules over an arbitrary field, and impose a condition we call $\Sigma$-cofibrancy on the operad. This condition also comes from [20, 16], and has of course been since used by other authors, too. It is automatically satisfied in characteristic 0 .

Finally, we would like to comment briefly on the relationship of our results with previous work, in particular the recent results of Hirsh and Millès [11]. The main concept of [11] is that of a properad, which is a variant of previous notions of PROPs [25] and dioperads [7]. PROPs, like operads, were first introduced in the context of infinite loop space theory [2]. PROP's (or dioperads) themselves are algebras over appropriate multisorted operads. A derived version of the concept of curvature of [11] is then equivalent to a special case of (a multisorted version of) D-structure. The use of the term curvature in this context was, in fact, coined by Getzler and Jones [8]. That paper provided the 
first clue why a notion of curvature may be Koszul dual to a unit. In some sense, [11] generalize that approach. While explicit comparisons with all existing work exceed the scope of the present paper, we do, in the Appendix, spell out the changes needed to treat the multisorted context, thus providing a framework for treating derived Koszul duality in all the known algebraic contexts.

It may be useful to make a brief remark on extensions of Koszul duality beyond operads: In these cases (e.g. PROP's), one typically discusses Koszul duality only for the PROP's themselves, but not for algebras over them. Such algebras may have both operations and cooperations (e.g. Hopf algebras), which causes them to behave badly from a categorical point of view. For example, the forgetful functor from such algebras into chain complexes typically is neither a left nor a right adjoint. For that reason, those types of algebras do not easily fit the Quillen framework [24], and what can be said about Koszul duality in this case remains largely an open problem.

The present paper is organized as follows: Because of the delicate technical nature of our construction, and the presence of a large number of variants of similar concepts in the literature, we found it necessary to make the present paper as self-contained as possible, even at the cost of redefining some known concepts, when there is ambiguity in them. In Section 2, we treat these necessary technical prerequisites. In Section 3. we treat rigorously the notion of a tree. For us, trees are ordered, or "planar" trees. Because of the sign issue, which is central to us, we also choose to be more pedantic and rigorous than is customary in this context. In Section 4, we review the Ginzburg-Kapranov bar construction in the setting we need, and implement the relevant sign devices. In Section 5, we introduce our version of the concept of a D-structure. In Section 6, we construct the derived category of D-structures, and prove that it is equivalent to the Quillen derived category of algebras

over the original operad. In the Appendix, we treat the multi-sorted case.

\section{PRELiminaries}

In this paper, we will work with unital operads $\mathcal{C}$ in the category $K$ Chain of chain complexes of $K$-modules where $K$ is a field. We will also call them $D G K$-module operads. This means a sequence $\mathcal{C}(n)$ of chain complexes of $K$-modules, together with an action of the permutation group $\Sigma_{n}$ on $\mathcal{C}(n), n=0,1,2, \ldots$ a unit chain map

$$
K \rightarrow \mathcal{C}(1)
$$


and an operation

$$
\begin{gathered}
\mathcal{C}\left(n_{1}\right) \otimes \cdots \otimes \mathcal{C}\left(n_{k}\right) \otimes \mathcal{C}(k) \rightarrow \mathcal{C}\left(n_{1}+\cdots+n_{k}\right), \\
x_{1} \otimes \cdots \otimes x_{k} \otimes x_{k+1} \mapsto \gamma\left(x_{1}, \ldots, x_{k} ; x_{k+1}\right)
\end{gathered}
$$

satisfying the usual equivariance, associativity and unit axioms modelled on the example $\mathcal{H}_{M}(n)=\operatorname{Hom}\left(M^{\otimes n}, M\right)$ where $M$ is a chain complex of Kmodules, and Hom denotes the internal Hom in the category of chain complexes of $K$-modules ([18]). As usual, when dealing with graded objects, we apply the Koszul sign

$$
(-1)^{j k}
$$

in the switch homomorphism between $x \otimes y$ and $y \otimes x$ for homogeneous elements $x, y$ of degrees $j, k$, respectively.

It may be more common to put the $\mathcal{C}(k)$ term first in the tensor product (1). We chose the current order of variables because we work in the context of trees, which we visualize as having roots in the bottom: When writing the trees in one line, it seems natural to write the upper parts of the tree to the left and the root to the right. It is, obviously, only a matter of signs.

It is also useful to introduce the operation

$$
\gamma_{j}: \mathcal{C}(k) \otimes \mathcal{C}(n) \rightarrow \mathcal{C}(n+k-1)
$$

given by

$$
\gamma_{j}(x, y)=\gamma(\underbrace{1, \ldots, 1}_{j-1 \text { times }}, x, \underbrace{1, \ldots, 1}_{n-j \text { times }}, y) .
$$

When considering non-unital operads (which we do not do in this paper, but which is, for example, the basic setup of [10]), one usually does include the operations $\gamma_{j}$ in the definition: the operation $\gamma$ can be recovered from them, but not vice versa.

A morphism of operads is a chain map which preserves the operations $\gamma$, the unit and the $\Sigma_{n}$-equivariances. By a $D G$-C-algebra $A$ we mean a homomorphism of operads

$$
\mathcal{C} \rightarrow \mathcal{H}_{A}
$$

This is equivalent data to having operations

$$
\theta: \underbrace{A \otimes \cdots \otimes A}_{n \text { times }} \otimes \mathcal{C}(n) \rightarrow A
$$

which satisfy the expected associativity, unit and equivariance conditions. Morphisms of $\mathcal{C}$-algebras are chain maps which preserve all the operations.

We will also use the notion of a monad, which is a generalization of an operad (in the sense that for every operad, there is an associated 
monad, and the algebras are the same). A monad in a category $Q$ is a functor $C: Q \rightarrow Q$ together with a product $\mu: C C \rightarrow C$ and a unit $\eta: I d \rightarrow C$ which satisfy associativity and unit axioms. A $C$-algebra $\mathrm{X}$ consists of a natural transformation $\theta: C X \rightarrow X$ which satisfies an associativity with respect to $\mu$ and a unit axiom with respect to $\eta$. Morphisms of $C$-algebras are morphisms in $Q$ which commute with the operation $\theta$ :

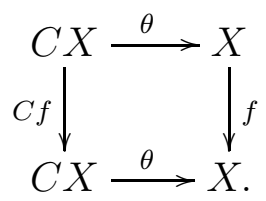

For an operad $\mathcal{C}$, there is a canonical monad $C$ such that $\mathcal{C}$-algebras are the same as $C$-algebras (they form canonically equivalent categories). The monad is given by

$$
C X=\bigoplus_{n \geq 0} X^{\otimes n} \otimes_{\Sigma_{n}} \mathcal{C}(n)
$$

where the symmetric group acts on $X^{\otimes n}$ by permutations (with the Koszul signs).

In the category of chain complexes of $K$-modules, by an equivalence we mean a chain map which induces an isomorphism in homology. If $X$ is a chain complex, $X[n]$ denotes the chain complex $X$ with degrees (in topology often referred to as dimensions) shifted by $n: X[n]_{k}=X_{k-n}$.

In important point is that for a general operad, when $f: X \rightarrow Y$ is an equivalence, $C f: C X \rightarrow C Y$ may not be an equivalence. In fact, this has less to do with the notion of an operad than with structure of chain complexes of $K\left[\Sigma_{n}\right]$-modules. To illustrate the problem, let $I\left[\Sigma_{n}\right]$ denote the chain complex of $K\left[\Sigma_{n}\right]$-modules where

$$
\begin{array}{cl}
I\left[\Sigma_{n}\right]_{i}= & K\left[\Sigma_{n}\right] \\
0 & \text { for } i=0,1 \\
0 & \text { else, }
\end{array}
$$

where the differential is the identity. Then we have an obvious chain map of $K\left[\Sigma_{n}\right]$-modules $\epsilon: K\left[\Sigma_{n}\right] \rightarrow I\left[\Sigma_{n}\right]$. (An ungraded module, when considered graded without further specification, is considered to be in dimension 0).

Suppose we are given chain complexes $X_{(k)}, k \in \mathbb{Z}_{\geq-1}$, where $X_{(-1)}=$ 0 , sets $I_{k}, k \in \mathbb{Z}_{>0}$, together with maps $q_{k}: I_{k} \rightarrow \mathbb{Z}$ and chain maps of $K\left[\Sigma_{n}\right]$-modules

$$
f_{k}: \bigoplus_{i \in I_{k}} K\left[\Sigma_{n}\right]\left[q_{k}(i)\right] \rightarrow X_{(k-1)}
$$


such that $X_{(k)}$ is a pushout of the diagram

$$
\begin{gathered}
\bigoplus_{i \in I_{k}} K\left[\Sigma_{n}\right]\left[q_{k}(i)\right] \stackrel{f_{k}}{\longrightarrow} X_{(k-1)} \\
\qquad \bigoplus \epsilon\left[q_{k}(i)\right] \\
\bigoplus_{i \in I_{k}} I\left[\Sigma_{k}\right]\left[q_{k}(i)\right] .
\end{gathered}
$$

A chain complex of $K\left[\Sigma_{n}\right]$-modules $X$ is called cell if it is of the form

$$
X \cong \operatorname{col} \lim _{k} X_{(k)}
$$

where $X_{(k)}$ are as above. (In particular, each $X_{(k)}$ is cell.)

We will call a chain complex of $K\left[\Sigma_{n}\right]$-modules $\Sigma$-cofibrant if it is a retract of a cell chain complex of $K\left[\sigma_{n}\right]$-modules. (Note that when $K$ is a field of characteristic 0, the assumption is automatically satisfied and hence vacuos.) We will call an DG $K$-module operad $\mathcal{C} \Sigma$-cofibrant if each $\mathcal{C}(n)$ is an $\Sigma$-cofibrant chain complex of $K\left[\Sigma_{n}\right]$-modules. The main point of considering these notions is the following

Proposition 1. Let $X$ be an $\Sigma$-cofibrant chain complex of $K\left[\Sigma_{n}\right]$ modules. Then the functor

$$
X \otimes_{K\left[\Sigma_{n}\right]} ?: K\left[\Sigma_{n}\right] \text {-modules } \rightarrow K\left[\Sigma_{n}\right] \text {-modules }
$$

preserves equivalences. Consequently, if $\mathcal{C}$ is an $\Sigma$-cofibrant $D G K$ module operad, then the associated monad

$C:$ chain complexes of $K$-modules $\rightarrow$ chain complexes of $K$-modules preserves equivalences.

\section{TREES}

In this section, we will rigorously define, and describe basic operations on what is usually referred to as "planar trees". Roughly speaking, they are (finite) rooted trees where the set of vertices which are immediately above each given vertex (i.e. are attached to it by an edge) come with a specified linear ordering. This determines a linear ordering on the entire set of vertices of the tree. In the context of the present paper, where there is need for extra sensitivity regarding signs, we felt compelled to be perhaps more rigorous about this concept than is usually customary, and write everything down "algebraically", without using pictures. In connection with this, we should, of course, note that mild variations in the concept are possible, for example, the ordering could be reversed. One variation which could be considered 
more substantial is that we distinguish between "leaves" and "non-leaf vertices of valency 0 ". This is because of the fact that we work with operads $\mathcal{C}$ and $\mathcal{C}$-algebras $A$ where we do not require $\mathcal{C}(0)=0$ : elements of $\mathcal{C}(0)$ are then attached to non-leaf vertices of valency 0 , while elements of a $\mathcal{C}$-algebra $A$ are attached to leaves.

Denote $\mathbf{n}=\{1, \ldots, n\},|S|$ for a (finite) set $S$ will denote the cardinality of $S$.

Definition 2. In this paper, a tree $(n, s, L)$ is the following data: a subset $L \subseteq \mathbf{n}$ (called the set of leaves) and map

$$
s: \mathbf{n}-\mathbf{1}(=\mathbf{n} \backslash\{n\}) \rightarrow \mathbf{n} \backslash L
$$

such that

(1) $s(x)>x$ for all $x$

(2) $x \leq y<s(x) \Rightarrow s(y) \leq s(x)$.

For $i \in \mathbf{n}$, the number $v_{s}(i)=\left|s^{-1}(i)\right|$ will be called the valency of $i$. Therefore, for $i \in L$, we have $v(i)=0$.

To interpret this definition in terms of usual planar trees, every $x$ is connected to $s(x)$ by an edge; the vertex $s(x)$ is "below" the vertex $x$ in the planar tree. Therefore, the root is the greatest element, $n$.

Lemma 3. Let $(n, s, L)$ be a tree, $n \notin L$ (note that $n \in L$ is only possible if $n=0$ ) and let

$$
\left\{k_{1}<k_{2}<\cdots<k_{m}\right\}=s^{-1}(n) .
$$

Then we have $k_{m}=n-1$. Putting $k_{0}=0, n_{i}=k_{i}-k_{i-1}, i=1, \ldots, m$, $\left(n_{i}, s_{i}, L_{i}\right)$ are trees where

$$
\begin{gathered}
s_{i}(j)=s\left(j+k_{i-1}\right)-k_{i-1}, \\
L_{i}=\left\{x-k_{i-1} \mid x \in L\right\} \cap \mathbf{n}_{\mathbf{i}} .
\end{gathered}
$$

We call $\left(n_{i}, s_{i}, L_{i}\right)_{i}$ the sequence of successor trees of $(n, s, L)$. Let $(n, s, L)$ be a tree with sequence of successor trees

$$
\left(n_{1}, s_{1}, L_{1}\right), \ldots\left(n_{m}, s_{m}, L_{m}\right) .
$$

Let $\sigma: \mathbf{m} \rightarrow \mathbf{m}$ be a permutation. Then there is a unique tree $\left(n, s^{\sigma}, L^{\sigma}\right)$ with sequence of successor trees

$$
\left(n_{\sigma(1)}, s_{\sigma(1)}, L_{\sigma(1)}\right), \ldots,\left(n_{\sigma(m)}, s_{\sigma(m)}, L_{\sigma(m)}\right) .
$$

Let $\sim$ be the smallest equivalence relation on trees such that 
(1) $(n, s, L) \sim\left(n, s^{\sigma}, L^{\sigma}\right)$ for any permutation $\sigma$ applicable.

(2) If $(n, s, L),\left(n^{\prime}, s^{\prime}, L^{\prime}\right)$ have sequences of successor trees

$$
\begin{gathered}
\left(n_{1}, s_{1}, L_{1}\right), \ldots\left(n_{m}, s_{m}, L_{m}\right), \\
\left(n_{1}, s_{1}^{\prime}, L_{1}^{\prime}\right), \ldots\left(n_{m}, s_{m}^{\prime}, L_{m}^{\prime}\right)
\end{gathered}
$$

where $\left(n_{i}, s_{i}, L_{i}\right) \sim\left(n_{i}, s_{i}^{\prime}, L_{i}^{\prime}\right), i=1, \ldots, m$, then

$$
(n, s, L) \sim\left(n^{\prime}, s^{\prime}, L^{\prime}\right) \text {. }
$$

Lemma 4. For trees $(n, s, L),\left(n, s^{\prime}, L^{\prime}\right)$, we have $(n, s, L) \sim\left(n, s^{\prime}, L^{\prime}\right)$ if and only if there exists a permutation $\sigma: \mathbf{n} \rightarrow \mathbf{n}$ such that $\sigma(L)=L^{\prime}$ and $s^{\prime}(\sigma(i))=\sigma(s(i))$ when applicable.

In the case of Lemma 4, we call $\sigma$ the intertwining permutation $(n, s, L) \rightarrow\left(n, s^{\prime}, L^{\prime}\right)$. Note that the permutation $\sigma$ may not be unique. Trees and intertwining permutations form a groupoid.

Next, we define edge contractions of trees. Let $(n, s, L)$ be a tree, $n \notin L$ with sequence of successor trees

$$
\left(n_{1}, s_{1}, L_{1}\right), \ldots,\left(n_{m}, s_{m}, L_{m}\right) .
$$

Choose $j \in \mathbf{m}$ with $n_{j} \notin L_{j}$ and let

$$
\left(p_{1}, t_{1}, M_{1}\right), \ldots\left(p_{q}, t_{q}, M_{q}\right)
$$

be the sequence of successor trees of $\left(n_{j}, s_{j}, L_{j}\right)$. Then there is a unique tree $\left(n-1, s_{j}^{\circ}, L_{j}^{\circ}\right)$ with sequence of successor trees

$$
\begin{aligned}
& \left(n_{1}, s_{1}, L_{1}\right), \ldots,\left(n_{j-1}, s_{j-1}, L_{j-1}\right), \\
& \left(p_{1}, t_{1}, M_{1}\right), \ldots,\left(p_{q}, t_{q}, M_{q}\right), \\
& \left(n_{j+1}, s_{j+1}, L_{j+1}\right), \ldots,\left(n_{m}, s_{m}, L_{m}\right) .
\end{aligned}
$$

We define a tree $\left(n-1, s^{\prime}, L^{\prime}\right)$ inductively to be an edge contraction of a tree $(n, s, L)$ if either

$$
\left(n-1, s^{\prime}, L^{\prime}\right)=\left(n-1, s_{j}^{\circ}, L_{j}^{\circ}\right)
$$

for some $j$, or $\left(n, s^{\prime}, L^{\prime}\right)$ has sequence of successor trees

$$
\begin{aligned}
& \left(n_{1}, s_{1}, L_{1}\right), \ldots,\left(n_{j-1}, s_{j-1}, L_{j-1}\right), \\
& \left(n_{j}-1, s_{j}^{\prime}, L_{j}^{\prime}\right), \\
& \left(n_{j+1}, s_{j+1}, L_{j+1}\right), \ldots,\left(n_{m}, s_{m}, L_{m}\right)
\end{aligned}
$$

where $\left(n_{j}-1, s_{j}^{\prime}, L_{j}^{\prime}\right)$ is an edge contraction of $\left(n_{j}, s_{j}, L_{j}\right)$. (The first case corresponds to contraction of an edge attached to the root, the second case corresponds to contractions of other edges.) 
Lemma 5. For $j \in(\mathbf{n}-\mathbf{1}) \backslash L$, there exists a unique edge contraction $\left(n-1, s^{\prime}, L^{\prime}\right)$ of the tree $(n, s, L)$ such that the map

$$
\tau=\tau_{s, j}:(\mathbf{n}-\mathbf{1}) \rightarrow \mathbf{n}
$$

given by

$$
\begin{aligned}
& \tau(k)=k \quad \text { for } k<j \\
& k+1 \text { for } k \geq j
\end{aligned}
$$

satisfies

$$
\begin{aligned}
& \tau\left(s^{\prime}(k)\right)=s \tau(k) \quad \text { if } s(k) \neq i \\
& s(i) \quad \text { if } s(k)=i \text {. }
\end{aligned}
$$

Moreover, every edge contraction is obtained in this way. We call ( $n-$ $\left.1, s^{\prime}, L^{\prime}\right)$ the edge contraction of $(n, s, L)$ at $j$.

We shall also use a particular left inverse $\rho=\rho_{s, j}$ of $\tau_{s, j}$ defined by

$$
\begin{gathered}
\tau \circ \rho=I d_{\mathbf{n}-\mathbf{1}}, \\
\rho(j):=s(j) .
\end{gathered}
$$

A tree $(n, s, L)$ is called a bush if $L=\mathbf{n}-\mathbf{1}$. (In some texts, this is called a corolla.) A tree $\left(n^{\prime}, s^{\prime}, L^{\prime}\right)$ is called a leaf contraction of a tree $(n, s, L)$ if there exist $1 \leq i \leq j \leq n, j \notin L$, such that

$$
s^{-1}(j)=\{i, i+1, \ldots, j-1\} \subseteq L,
$$

$n^{\prime}=n-j+i$ and the map

$$
\tau=\tau_{s, i, j}: \mathbf{n}^{\prime} \rightarrow \mathbf{n}
$$

given by

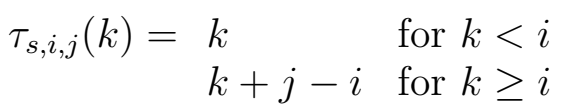

satisfies

$$
\begin{gathered}
\tau\left(s^{\prime}(k)\right)=s(\tau(k)) \text { for all } k \in \mathbf{n}^{\prime}, \\
L^{\prime}=\tau^{-1}(L) \cup\{i\} .
\end{gathered}
$$

We then call $\left(n^{\prime}, s^{\prime}, L^{\prime}\right)$ the leaf contraction of $(n, s, L)$ at $(i, j)$. Pictorially, this corresponds to contracting a bush of all leaves attached to a vertex, which has no other successor trees, to a single point. Again, we shall also use a left inverse $\rho=\rho_{s, i, j}$ of $\tau_{s, i, j}$ where

$$
\begin{gathered}
\rho \circ \tau=I d_{\mathbf{n}^{\prime}}, \\
\rho(k):=i \text { for } k \in\{i, i+1, \ldots, j-1\} .
\end{gathered}
$$


Lemma 6. Let $\sigma: \mathbf{n} \rightarrow \mathbf{n}$ be an intertwining permutation

$$
\sigma:(n, s, L) \rightarrow(n, t, M) \text {. }
$$

1. Let $j \in(\mathbf{n}-\mathbf{1}) \backslash L$ Let $\left(n-1, s^{\prime}, L^{\prime}\right)$ resp. $\left(n-1, t^{\prime}, M^{\prime}\right)$ be the edge contractions of $(n, s, L)$ resp. $(n, t, M)$ at $j$ resp. $\sigma(j)$. Then the unique permutation

$$
\sigma^{\prime}: \mathbf{n}-\mathbf{1} \rightarrow \mathbf{n}-\mathbf{1}
$$

with

$$
\tau_{t, \sigma(j)} \sigma^{\prime}=\sigma \tau_{s, j}
$$

intertwines

$$
\sigma^{\prime}:\left(n-1, s^{\prime}, L^{\prime}\right) \rightarrow\left(n-1, t^{\prime}, M^{\prime}\right) .
$$

2. Let $\left(n^{\prime}, s^{\prime}, L^{\prime}\right)$ be a leaf contraction of $(n, s, L)$ at $(i, j)$. Then there exists a leaf contraction $\left(n^{\prime}, t^{\prime}, M^{\prime}\right)$ at $(\sigma(j)-j+i, \sigma(j))$ and the unique permutation

$$
\sigma^{\prime}: \mathbf{n}^{\prime} \rightarrow \mathbf{n}^{\prime}
$$

which satisfies

$$
\tau_{t, \sigma(j)-k+i} \sigma^{\prime}=\sigma \tau_{s, i, j}
$$

intertwines

$$
\sigma^{\prime}:\left(n^{\prime}, s^{\prime}, L^{\prime}\right) \rightarrow\left(n^{\prime}, t^{\prime}, M^{\prime}\right)
$$

\section{The augmented Ginzburg-Kapranov Bar Construction}

An explanation of signs: Signs are well known to cause pains throughout algebraic topology. Traditionally, one deals with this by writing terms in certain order, and attaching a specific sign convention when order of elements is exchanged. The Koszul-Milnor signs, where an exchange of elements of degree $k$ and $\ell$ introduces a sign of $(-1)^{k \ell}$, is well known. Still, in a more complicated setting, such as constructions indexed by trees, with multiple operations and differentials, sign conventions become complicated and their consistency is difficult to verify. Ginzburg and Kapranov [10] introduced a very elegant solution, making their sign an element of a "determinant line" on a space generated by the edges. Tensoring with this line, and interpreting operators contracting lines as left differentiations eliminates the need to discuss signs explicitly, as the determinant line is "functorial", and it is therefore immediate that omitting two lines in opposite order always reverses signs, no matter where the corresponding variables are written (by the functoriality, they may as well be written, say, in the first two places). 
While the present paper was originally written with explicit signs depending on the order of elements, and we had all the sign consistencies checked, the signs seemed artificial and not illuminating. Additionally, it was difficult to be honestly sure that there is no hidden mistake. Because of that, we very much wanted to use the Ginzburg-Kapranov determinant line for signs attached to contractions of edges, but there was one problem: The Ginzburg-Kapranov determinant does not handle differential graded objects with their own internal differential, since those require their own sign scheme (such as the Koszul-Milnor signs), and we must also express how both of the two kinds of signs are compatible.

Because of that, we searched for an appropriate receptacle line for the internal sign of a tensor product of differential graded objects: we require naturality with respect to change of order of tensor factors, merging two factors by a "graded-commutative" product, and anticommutation of internal differentials on two different tensor factors. An exterior algebra is clearly not appropriate for this purpose if we want the internal differential to be accompanied by a simple operator such as multiplication by a generator corresponding to the given tensor factor (or, alternately, differentiation - we ended up using multiplication). While the internal differential satisfies $d d=0$ on elements, this is not true on degrees: One must be able to switch back and forth between even and odd degrees using the same operator.

We then realized that these desiderata pretty much uniquely specify the moduls of monomials in a Clifford algebra, where the square of the sign element corresponding to each tensor factor is 1: one has the correct commutation relations, while the sign of an internal differential in a given factor is expressed by multiplication by that factor. It is then plain to see that two internal differentials of different tensor factors automatically anti-commute, eliminating lengthy and artificial verifications. Formally, the sign-holding objects we introduce are as follows:

For a tree $(n, s, L)$ and $i \in \mathbf{n}$, denote

$$
\epsilon(i)=\epsilon_{(n, s, L)}(i)=|\mathbf{i} \backslash L| .
$$

Let $K$ be a field and let $\mathcal{C}$ be an $\Sigma$-cofibrant operad in the category of chain complexes of $K$-modules (see the definitions in Section 2 above). Let $A$ be a $\mathcal{C}$-algebra in the category $K$-Chain (again, see Section 2 ). We let

$$
\Lambda(n, s, L)
$$


be a $K$-valued exterior algebra on indeterminates $e_{i}, i \in \mathbf{n}-\mathbf{1} \backslash L$. Let

$$
C_{n}
$$

be a Clifford algebra on indeterminates $f_{i}, i \in \mathbf{n}$, by which we mean the factor algebra of the tensor algebra on the $f_{i}$ 's by the relations

$$
f_{i}^{2}=1, f_{i} f_{j}=-f_{j} f_{i} \text { for } i \neq j
$$

(Although the elements $e_{i}, j_{j}$ carry sign information, we consider them to be in degree 0 .) We also introduce a $K$-algebra structure on

$$
\Lambda(n, s, L) \otimes C_{n}
$$

by setting

$$
f_{j} \cdot e_{i}=-e_{i} \cdot f_{j}
$$

We shall write

$$
\operatorname{Det}(n, s, L)
$$

for the top degree summand of $\Lambda(n, s, L)$, i.e. the sub- $K$-module of $\Lambda(n, s, L)$ spanned by

$$
\prod_{i \in(\mathbf{n}-\mathbf{1}) \backslash L} e_{i}
$$

For $\epsilon=\left(\epsilon_{1}, \ldots, \epsilon_{n}\right) \in(\mathbb{Z} / 2)^{n}$, we denote by $C_{n, \epsilon}$ the sub- $K$-module of $C_{n}$ spanned by

$$
f_{1}^{\epsilon_{1}} \ldots f_{n}^{\epsilon_{n}} .
$$

For $\varepsilon \in \mathbb{Z} / 2$, and a graded $K$-module $M$, denote by $M_{\varepsilon}$ the submodule spanned by all homogeneous elements of degrees equal to $\varepsilon \bmod 2$.

The augmented bar construction $\widetilde{B}_{\mathcal{C}}(A)$ is defined by

$$
\begin{aligned}
& \widetilde{B}_{\mathcal{C}}(A)= \\
& \left(\bigoplus_{(n, s, L)} \bigoplus_{\epsilon \in(\mathbb{Z} / 2)^{n}} \operatorname{Det}(n, s, L) \otimes\right. \\
& \left.C_{\epsilon} \otimes\left(\bigotimes_{i \in L} A_{\epsilon_{i}} \otimes \bigotimes_{\substack{i \in \mathbf{n} \backslash L \\
v(i)=k}} \mathcal{C}(k)_{\epsilon_{i}}\right)\left[\epsilon_{(n, s, L)}(n)\right]\right) / \sim .
\end{aligned}
$$

The direct sum is over all trees. The equivalence is the smallest congruence of $K$-modules satisfying, for

$$
\sigma:(n, s, L) \rightarrow\left(n, s^{\prime}, L^{\prime}\right)
$$




$$
\begin{aligned}
& \prod_{i} e_{i} \prod_{j} f_{j} \cdot \bigotimes_{i \in L}\left(x_{i} \in A\right) \otimes \bigotimes_{\substack{i \in \mathbf{n} \backslash L \\
v_{s}(i)=k}}\left(x_{i} \in \mathcal{C}(k)\right) \\
& \sim \prod_{i} e_{\sigma(i)} \prod_{j} f_{\sigma(j)} \cdot \bigotimes_{i \in L^{\prime}}\left(y_{i} \in A\right) \otimes{\substack{i \in \mathbf{n} \backslash L^{\prime} \\
v_{s^{\prime}}(i)=k}}_{\bigotimes}\left(y_{i} \in \mathcal{C}(k)\right) .
\end{aligned}
$$

Note: since the algebras $\Lambda(n, s, L), C_{n}$ are not commutative, the products are taken in the order of indexing. The congruence relation identifies the summands corresponding to equivalent trees in the sense of Section 3, i.e. with respect to permuting successor trees of a given vertex, with the appropriate signs (note that permuting the exterior resp. Clifford generators introduces the sign associated with putting them back in the original order).

The differential $d$ on $\widetilde{B}_{\mathcal{C}}(A)$ is the sum of the following kinds of maps:

(1) The edge contraction summands. If $\left(n-1, s^{\prime}, L^{\prime}\right)$ is an edge contraction of $(n, s, L)$ at $q$, let

$$
\lambda_{\rho}: \Lambda(n, s, L) \rightarrow \Lambda\left(n-1, s^{\prime}, L^{\prime}\right)
$$

be given by

$$
e_{i} \mapsto e_{\rho(i)},
$$

and let

$$
c_{\rho}: C_{n} \mapsto C_{n-1}
$$

be defined by

$$
f_{j} \mapsto f_{\rho(j)}
$$

Then we have

$$
\begin{aligned}
& \prod_{i} e_{i} \prod_{j} f_{j} \cdot \bigotimes_{i \in L}\left(x_{i} \in A\right) \otimes \bigotimes_{i \in \mathbf{n} \backslash L}\left(x_{i} \in \mathcal{C}(k)\right) \\
& v_{s}(i)=k \\
& \mapsto \lambda_{\rho}\left(\frac{\partial}{\partial e_{j}} \prod_{i} e_{i}\right) c_{\rho}\left(\prod_{j} f_{j}\right) \cdot \bigotimes_{i \in L^{\prime}}\left(y_{i} \in A\right) \otimes{\substack{i \in(\mathbf{n}-\mathbf{1}) \backslash L^{\prime} \\
v_{s^{\prime}}(i)=\ell}}_{L^{\prime}}\left(y_{i} \in \mathcal{C}(\ell)\right)
\end{aligned}
$$

where

$$
y_{i}=x_{\tau_{s, j}(i)} \text { if } i \neq s(q)-1,
$$

and

$$
y_{s(q)-1}=\gamma_{\ell}\left(x_{q}, x_{s_{q}}\right)
$$


if

$$
s^{-1}(s(q))=\left\{q_{1}<\cdots<q_{m}\right\}, q_{\ell}=q .
$$

(2) The leaf contraction summands. If $\left(n^{\prime}, s^{\prime}, L^{\prime}\right)$ is a leaf contraction of $(n, s, L)$ at $(p, r)$, let, again,

$$
\lambda_{\rho}: \Lambda(n, s, L) \rightarrow \Lambda\left(n^{\prime}, s^{\prime}, L^{\prime}\right)
$$

be given by

$$
e_{i} \mapsto e_{\rho(i)}
$$

and let

$$
c_{\rho}: C_{n} \mapsto C_{n^{\prime}}
$$

be defined by

$$
f_{j} \mapsto f_{\rho(j)}
$$

$$
\prod_{i} e_{i} \prod_{j} f_{j} \cdot \bigotimes_{q \in L}\left(x_{q} \in A\right) \otimes \bigotimes_{\substack{q \in \mathbf{n} \backslash L \\ v_{s}(q)=k}}^{\bigotimes}\left(x_{q} \in \mathcal{C}(k)\right)
$$

$$
\mapsto \lambda_{\rho}\left(\frac{\partial}{\partial e_{j}} \prod_{i} e_{i}\right) c_{\rho}\left(\prod_{j} f_{j}\right) \cdot \bigotimes_{q \in L^{\prime}}\left(y_{q} \in A\right) \otimes \underset{\substack{q \in\left(\mathbf{n}^{\prime}\right) \backslash L^{\prime} \\ v_{s^{\prime}}(q)=\ell}}{\bigotimes}\left(y_{q} \in \mathcal{C}(\ell)\right)
$$

where

$$
\begin{gathered}
y_{q}=x_{\tau_{s, i, j}(q)} \text { if } q \neq r, \\
y_{p}=\theta\left(x_{p}, \ldots, x_{r-1} ; x_{r}\right) .
\end{gathered}
$$

(3) The internal differential summands. Denoting by $\partial$ the internal differential on $A$ and $\mathcal{C}(k)$ for each $i \in \mathbf{n}$, we have

$$
\begin{gathered}
\prod_{\ell} e_{\ell} \prod_{j} f_{j} \cdot \bigotimes_{q \in L}\left(x_{q} \in A\right) \otimes \underbrace{\bigotimes}_{\substack{q \in \mathbf{n} \backslash L \\
v_{s}(q)=k}}\left(x_{q} \in \mathcal{C}(k)\right) \\
\mapsto f_{i} \prod_{\ell} e_{\ell} \prod_{j} f_{j} \cdot \bigotimes_{q \in L}\left(y_{q} \in A\right) \otimes \bigotimes_{\substack{q \in(\mathbf{n}) \backslash L \\
v_{s}(q)=k}}^{\bigotimes}\left(y_{q} \in \mathcal{C}(k)\right)
\end{gathered}
$$

where

$$
\begin{gathered}
y_{q}=x_{q} \text { for } q \neq i, \\
y_{i}=\partial\left(x_{i}\right) .
\end{gathered}
$$


One readily verifies that the sum $d$ of all these maps $\widetilde{B}_{\mathcal{C}}(A) \rightarrow \widetilde{B}_{\mathcal{C}}(A)$ does, in fact, satisfy

$$
d \circ d=0 .
$$

This, in effect, follows from the anticommutation of the operations $\partial / \partial e_{i}$, and left multiplication by the generators $f_{j}$.

\section{Proposition 7.}

$$
H\left(\widetilde{B}_{\mathcal{C}}(A), d\right)=0 .
$$

Proof. Define, for a tree $(n, s, L)$, a tree $(n+1, \bar{s}, L)$ by

$$
\begin{gathered}
\bar{s}(i)=s(i) \text { for } i<n, \\
\bar{s}(n)=n+1 .
\end{gathered}
$$

Define a map of degree 1

$$
h: \widetilde{B}_{\mathcal{C}}(A) \rightarrow \widetilde{B}_{\mathcal{C}}(A)
$$

by

$$
\begin{aligned}
& \prod_{i} e_{i} \cdot \prod_{j} f_{j} \cdot \bigotimes_{j \in L}\left(x_{j} \in A\right) \otimes \bigotimes_{j \in \mathbf{n} \backslash L}\left(x_{j} \in \mathcal{C}(k)\right) \\
& v_{s}(j)=k \\
& \mapsto e_{n} \cdot \prod_{i} e_{i} \cdot \prod_{j} f_{j} \cdot \bigotimes_{j \in \bar{L}}\left(y_{j} \in A\right) \otimes \bigotimes_{j \in(\mathbf{n}+\mathbf{1}) \backslash L}\left(y_{j} \in \mathcal{C}(k)\right) \\
& v_{\bar{s}}(j)=k
\end{aligned}
$$

where

$$
\begin{gathered}
y_{j}=x_{j} \text { for } j \in \mathbf{n}, \\
y_{n+1}=1 .
\end{gathered}
$$

(Note that the tree $(n+1, \bar{s}, \bar{L})$ is obtained from $(n, s, L)$ by "grafting", i.e. by attaching a new root below the old root, and connecting them with an edge. The labels of the "grafter tree" stay the same, the label of the new root is $n+1$.)

Then

$$
d h+h d=I d .
$$


Let

$$
A \stackrel{\subseteq}{\longrightarrow} \widetilde{B}_{\mathcal{C}}(A)
$$

$$
a \longmapsto a \in \bigoplus_{(1, *,\{1\})} A
$$

Denote

$$
B_{\mathcal{C}}(A):=\left(\widetilde{B}_{\mathcal{C}}(A) / A\right)[-1]
$$

Then there is a map

$$
\mu: B_{\mathcal{C}}(A) \rightarrow A
$$

given by

$$
\begin{aligned}
& \bigotimes_{j \in L}\left(x_{j} \in A\right) \otimes \bigotimes_{\substack{j \in \mathbf{n} \backslash L \\
v_{s}(j)=k}}^{\bigotimes}\left(x_{j} \in \mathcal{C}(k)\right) \\
& \mapsto \theta\left(x_{1}, \ldots, x_{n-1} ; x_{n}\right) \text { if }(n, s, L) \text { is a bush } \\
& \mapsto 0 \text { else. }
\end{aligned}
$$

Proposition 8. (1) The map (7) is a chain map, and an equivalence for an $\Sigma$-cofibrant $D G K$-module operad $\mathcal{C}$.

(2) There is a natural DG $\mathcal{C}$-algebra structure on $B_{\mathcal{C}}(A)$ such that (7) is a map of $D G \mathcal{C}$-algebras.

Proof. For (1), to prove that (7) is a chain map, it suffices to prove that it vanishes on differentials of trees $(n, s, L)$ where $\epsilon_{(n, s, L)}(n)=2$. On such trees, however, the differential has two summands (one edge contraction and one leaf contraction), which cancel after applying (7) to them.

By definition, further, (77) fits into the following diagram of chain complexes:

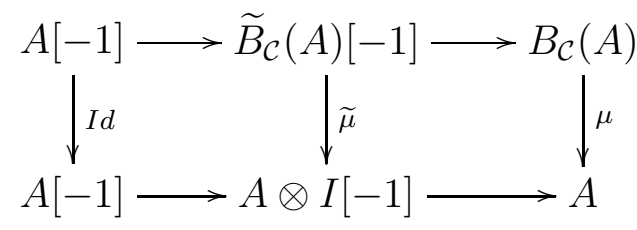

where $I$ is the chain complex of $K$-modules

$$
K \stackrel{\cong}{\longrightarrow} K
$$


in dimensions 1,0 .

The map $\tilde{\mu}$ has

$$
a \in \bigoplus_{(1, *,\{1\})} A \mapsto a .
$$

Since the source and target of $\widetilde{\mu}$ are both acyclic, $\widetilde{\mu}$ is an equivalence, so $\mu$ is an equivalence by (8) and the 5-lemma.

The $\mathcal{C}$-algebra structure on $B_{\mathcal{C}}(A)$ is given by

$$
\begin{aligned}
& \prod_{i} e_{i, q} \prod_{j<n_{q}} f_{j, q} \cdot \prod_{q=1}^{m} f n_{q}, q \cdot \\
& \bigotimes_{q=1}^{m}\left(\bigotimes_{j \in L_{q}}\left(x_{j, q} \in A\right) \otimes \underset{\substack{j \in \mathbf{n}_{\mathbf{q}} \backslash L_{q} \\
v_{s}(j)=k}}{\bigotimes}\left(x_{j, q} \in \mathcal{C}(k)\right)\right. \\
& \mapsto \prod_{q}(\prod_{i} e_{\ell_{q}(i)} \cdot \prod_{j<n_{q}} f_{\ell_{q}(j)} \cdot f_{n} \cdot \bigotimes_{j \in L}\left(y_{j} \in A\right) \otimes \underbrace{\bigotimes}_{\substack{j \in \mathbf{n} \backslash L \\
v_{s}(j)=k}}\left(y_{j} \in \mathcal{C}(k)\right)
\end{aligned}
$$

where the left had side indicates a typical element of

$$
\left(B_{\mathcal{C}}(A)\right)^{\otimes m} \otimes \mathcal{C}(m),
$$

with $e_{i, q}, f_{j, q}$ denoting the exterior and Clifford elements assigned to the $q$ 'th $B_{\mathcal{C}}(A)$ factor, $\left(n_{q}, s_{q}, L_{q}\right)$ are successor trees of a tree $(n, s, L)$, and we write

$$
\begin{gathered}
\ell_{q}(i)=i+\left(n_{1}-1\right)+\cdots+\left(n_{q-1}-1\right), \\
n=\sum_{q=1}^{m} n_{q}-m+1,
\end{gathered}
$$

for $s_{q}(j) \neq n_{q}$,

$$
s\left(\ell_{q}(j)\right)=\ell_{q}\left(s_{q}(j)\right) \text {, }
$$

for $s_{q}(j)=n_{q}$,

$$
s\left(\ell_{q}(j)\right)=n,
$$

for $1 \leq j<n_{q}$

$$
\begin{gathered}
y_{\ell_{1}(j)}=x_{j, q}, \\
y_{n}=\gamma\left(x_{n, 1}, \ldots, x_{n, q} ; x\right) .
\end{gathered}
$$




\section{D-Structures}

Proposition 9. There exists a graded homomorphism of $K$-modules

$$
\bigoplus_{n \geq 0} \delta_{n}=\delta: \widetilde{B}_{\mathcal{C}}(A) \rightarrow \bigoplus_{n \geq 0} \widetilde{B}_{\mathcal{C}}(A)^{\otimes n} \otimes_{\Sigma_{n}} \mathcal{C}(n)=B_{\mathcal{C}}(A)
$$

such that if we denote, for $x \in \widetilde{B}_{\mathcal{C}}(A)$,

$$
\left.\delta(x)=\sum\left(\delta_{n}^{\prime}(x)\right)_{1} \otimes \cdots \otimes\left(\delta_{n}^{\prime}(x)\right)_{n} \otimes \delta_{n}^{\prime \prime}(x)\right)
$$

with $\left(\delta_{n}^{\prime}(x)\right)_{i} \in \widetilde{B}_{\mathcal{C}}(A), \delta_{n}^{\prime \prime}(x) \in \mathcal{C}(n)$, then

$$
\begin{aligned}
& d\left(x_{1} \otimes \cdots \otimes x_{n} \otimes x_{n+1}\right) \\
& =\sum_{i=1}^{n+1}(-1)^{\left|x_{1}\right|+\ldots\left|x_{i-1}\right|} x_{1} \otimes \cdots \otimes x_{i-1} \otimes d x_{i} \otimes x_{i+1} \otimes \cdots \otimes x_{n+1} \\
& +\sum_{i=1}^{n} \sum_{m \geq 0}(-1)^{\left|x_{1}\right|+\cdots+\left|x_{i}\right|+\left|\delta_{m}^{\prime \prime}\left(x_{i}\right)\right| \cdot\left(\left|x_{i+1}\right|+\cdots+\left|x_{n}\right|\right)} x_{1} \otimes \ldots \\
& \ldots x_{i} \otimes\left(\delta_{m}^{\prime}\left(x_{i}\right)\right)_{1} \otimes \cdots \otimes\left(\delta_{m}^{\prime}\left(x_{i}\right)\right)_{m} \otimes x_{i+1} \otimes \ldots \\
& \cdots \otimes x_{n} \otimes \gamma_{i}\left(\delta_{n}^{\prime \prime}\left(x_{i}\right), x_{n+1}\right) .
\end{aligned}
$$

Proof. For an element $x \in \widetilde{B}_{\mathcal{C}}(A)$ indexed over a tree of the form $(1, *,\{1\})$ (thus, $x \in A)$, we put

$$
\delta(x)=0 .
$$

For elements indexed over a tree $(n, s, L)$ with $n \notin L$ with successor trees $\left(n_{i}, s_{i}, L_{i}\right), i=1, \ldots, k$ (note that the case $n=1$ may occur, in which case we have $k=0$ ), define

$$
\begin{aligned}
& \delta\left(\prod_{\ell} e_{\ell} \cdot \prod_{j} f_{j} \cdot \bigotimes_{j \in L}\left(x_{j} \in A\right) \otimes \bigotimes_{\substack{j \in \mathbf{n} \backslash L \\
v_{s}(j)=k}}\left(x_{j} \in \mathcal{C}(k)\right)\right) \\
& =\prod_{\ell} e_{\ell} \cdot \prod_{j} f_{j} \cdot \bigotimes_{i=1}^{k}\left(\bigotimes_{j=n_{1}+\cdots+n_{i-1}+1}^{n_{1}+\cdots+n_{i}} x_{j}\right) \otimes\left(x_{n} \in \mathcal{C}(k)\right) .
\end{aligned}
$$

Note carefully that this formula hides a sign coming from the shuffle of the $e_{\ell}$ 's and $f_{j}$ 's so that the variables corresponding to the individual 
$\widetilde{B}_{\mathcal{C}}(A)$ factors on the right hand side are moved together. The sign in the second summand of the differential comes from the fact that the root of the trees corresponding to the $i$ 'th factor must be moved to the right to apply the operad operation.

Definition 10. Let $(\mathcal{C}, \partial)$ be a unital $\Sigma$-cofibrant DG-operad over a field $K$. Define

$$
C N=\bigoplus_{n \geq 0} N^{\otimes n} \otimes_{\Sigma_{n}} \mathcal{C}(n)
$$

A $D$-structure with respect to $\mathcal{C}$ is a DG- $K$-module $(N, d)$ together with a graded homomorphism of $K$-modules

$$
\begin{gathered}
\delta: N \rightarrow C N, \\
x \mapsto \sum_{n}\left(\left(\delta_{n}^{\prime}(x)\right)_{1} \otimes \cdots \otimes\left(\delta_{n}^{\prime}(x)\right)_{n} \otimes \delta_{n}^{\prime \prime}(x)\right),
\end{gathered}
$$

such that the map $\Delta: C N \rightarrow C N$ given by

$$
\begin{aligned}
& \Delta\left(x_{1} \otimes \cdots \otimes x_{n} \otimes x_{n+1}\right) \\
& =\sum_{i=1}^{n+1}(-1)^{\left|x_{1}\right|+\ldots\left|x_{i-1}\right|} x_{1} \otimes \cdots \otimes x_{i-1} \otimes d x_{i} \otimes x_{i+1} \otimes \cdots \otimes x_{n+1} \\
& +\sum_{i=1}^{n} \sum_{m \geq 0}(-1)^{\left|x_{1}\right|+\cdots+\left|x_{i}\right|+\left|\delta_{m}^{\prime \prime}\left(x_{i}\right)\right| \cdot\left(\left|x_{i+1}\right|+\cdots+\left|x_{n}\right|\right)} x_{1} \otimes \ldots \\
& \ldots x_{i} \otimes\left(\delta_{m}^{\prime}\left(x_{i}\right)\right)_{1} \otimes \cdots \otimes\left(\delta_{m}^{\prime}\left(x_{i}\right)\right)_{m} \otimes x_{i+1} \otimes \ldots \\
& \cdots \otimes x_{n} \otimes \gamma_{i}\left(\delta_{n}^{\prime \prime}\left(x_{i}\right), x_{n+1}\right) .
\end{aligned}
$$

defines a differential on $C N$ (i.e., $\Delta \circ \Delta=0$ ), and furthermore, $(C N, \Delta)$, with the $\mathcal{C}$-algebra structure on $C N$ in the category of graded $K$ modules coming from the monad, is a DG-C-algebra.

For our purposes, the concept of a D-structure is motivated by the following Proposition. In the case of the operad defining $A$-modules over an algebra $A$, it was also used in [17]. For further motivation, see Comment 2 before Definition 13 below.

Proposition 11. $\left(\widetilde{B}_{\mathcal{C}}(A), \delta\right)$ is a $\mathcal{C}$-D-structure.

Proof. This follows immediately from Proposition 9. 
Comment: Denote, for a $\mathcal{C}$-D-structure $(N, \Delta)$, by $C_{\Delta}(N)$ the DG$\mathcal{C}$-algebra $(C N, \Delta)$. Note that the explicit notation is justified, as $C N$ has its own $\mathcal{C}$-algebra structure coming from the fact that $C$ is the monad defining $\mathcal{C}$-algebras. Recall also that the unit $\eta: N \rightarrow C N$ is a chain map.

The map $\eta: N \rightarrow C_{\Delta}(N)$, on the other hand, is not a chain map. In fact, it is easily seen that the definition implies

$$
\eta d+\delta=\Delta \eta
$$

which in turn implies that

$$
x \mapsto(-1)^{|x|} \delta(x)
$$

is a chain map $\delta^{\prime}: N \rightarrow C_{\Delta}(N)$. Further, this map is null-homotopic by (10). In the case of $N=\widetilde{B}_{\mathcal{C}}(A), \delta^{\prime}$ is the projection

$$
\widetilde{B}_{\mathcal{C}}(A) \rightarrow B_{\mathcal{C}}(A)[1]
$$

of (6). In view of Proposition 7, then, one may ask if every D-structure has 0 homology. We will see that this is not the case. (By Comment 2 after Definition 13, the differential $d$ on $N$ can be 0 .)

Definition 12. A morphism of $\mathcal{C}$-D-structures $f:(N, \Delta) \rightarrow\left(N^{\prime}, \Delta^{\prime}\right)$ is a homomorphism of graded $K$-modules

$$
f_{0}: N \rightarrow C_{\Delta^{\prime}}\left(N^{\prime}\right)
$$

which extends to a homomorphism of DG-C-algebras $\bar{f}: C_{\Delta}(N) \rightarrow$ $C_{\Delta^{\prime}}\left(N^{\prime}\right)$ in the sense of the following diagram:

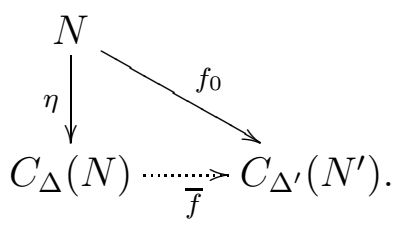

The identity morphism is $\eta$. For $g:\left(N^{\prime}, \Delta^{\prime}\right) \rightarrow\left(N^{\prime \prime}, \Delta^{\prime \prime}\right)$, composition is defined by

$$
(g \circ f)_{0}=\bar{g} \bar{f} \eta
$$

Comments: 1 . Note that $\bar{f}$ is uniquely determined as a map of $\mathcal{C}$ algebras because $C_{\Delta}(N)$ is equal to $C N$ as a graded $K$-module and $\eta: N \rightarrow C N$ is a universal homomorphism of graded $K$-modules into $\mathcal{C}$-algebras in the category of graded $K$-modules. Thus, the condition on $f_{0}$ reduces to requiring that $\bar{f}$ be a chain map. This can be written explicitly as follows: For

$$
x_{1} \otimes \cdots \otimes x_{n} \otimes x_{n+1} \in N^{\otimes n} \otimes \mathcal{C}(n),
$$


one requires that

$$
\begin{aligned}
& \sum_{i=1}^{n}(-1)^{\left|x_{1}\right|+\ldots\left|x_{i-1}\right|} f_{0}\left(x_{1}\right) \otimes \cdots \otimes f_{0}\left(x_{i-1}\right) \otimes d f_{0}\left(x_{i}\right) \otimes f_{0}\left(x_{i+1}\right) \otimes \cdots \otimes f_{0}\left(x_{n}\right) \otimes x_{n+1} \\
& +\sum_{i=1}^{n} \sum_{m \geq 0}(-1)^{\left|x_{1}\right|+\cdots+\left|x_{i}\right|+\left|\delta_{m}^{\prime \prime}\left(x_{i}\right)\right| \cdot\left(\left|x_{i+1}\right|+\cdots+\left|x_{n}\right|\right)} f_{0}\left(x_{1}\right) \otimes \ldots \\
& \ldots f_{0}\left(x_{i}\right) \otimes\left(\delta_{m}^{\prime}\left(f_{0}\left(x_{i}\right)\right)\right)_{1} \otimes \cdots \otimes\left(\delta_{m}^{\prime}\left(f_{0}\left(x_{i}\right)\right)\right)_{m} \otimes f_{0}\left(x_{i+1}\right) \otimes \ldots \\
& \ldots \otimes f_{0}\left(x_{n}\right) \otimes \gamma_{i}\left(\delta_{n}^{\prime \prime}\left(f_{0}\left(x_{i}\right)\right), x_{n+1}\right) \\
& =\sum_{i=1}^{n}(-1)^{\left|x_{1}\right|+\ldots\left|x_{i-1}\right|} f_{0}\left(x_{1}\right) \otimes \cdots \otimes f_{0}\left(x_{i-1}\right) \otimes f_{0}\left(d x_{i}\right) \otimes f_{0}\left(x_{i+1}\right) \otimes \cdots \otimes f_{0}\left(x_{n}\right) \otimes x_{n+1} \\
& +\sum_{i=1}^{n} \sum_{m \geq 0}(-1)^{\left|x_{1}\right|+\cdots+\left|x_{i}\right|+\left|\delta_{m}^{\prime \prime}\left(x_{i}\right)\right| \cdot\left(\left|x_{i+1}\right|+\cdots+\left|x_{n}\right|\right)} f_{0}\left(x_{1}\right) \otimes \ldots \\
& \ldots f_{0}\left(x_{i}\right) \otimes f_{0}\left(\left(\delta_{m}^{\prime}\left(x_{i}\right)\right)_{1}\right) \otimes \cdots \otimes f_{0}\left(\left(\delta_{m}^{\prime}\left(x_{i}\right)\right)_{m}\right) \otimes f_{0}\left(x_{i+1}\right) \otimes \ldots \\
& \ldots \otimes f_{0}\left(x_{n}\right) \otimes \gamma_{i}\left(\delta_{n}^{\prime \prime}\left(x_{i}\right), x_{n+1}\right) .
\end{aligned}
$$

2. It follows immediately from (9) and (10) that the category of $\mathcal{C}$ - $D$ structures is in fact equivalent to the full subcategory of the category of $\mathcal{C}$-algebras on objects which are free $\mathcal{C}$-algebras after forgetting differentials. Such objects are called quasi-free or almost free algebras and were introducted, in the case of augmented unital operads, by Getzler and Jones [9] and further investigated by Fresse [6]. Using this, one can deduce the augmented case of the results of the present paper from [9, 6]. The authors do not know if this was historically recognized as a restatement of non-unital (or, equivalently, augmented unital) Koszul duality. Definition 10 generalizes the form in which $D$-structures first arose in geometry [17], where it was also first observed that, at least in that special case, augmentation is not needed to make the arguments, and that this is unital Koszul duality.

Definition 13. A morphism $f:(N, \Delta) \rightarrow\left(N^{\prime}, \Delta^{\prime}\right)$ of $\mathcal{C}$-D-structures is an equivalence if the homomorphism $\bar{f}: C_{\Delta}(N) \rightarrow C_{\Delta^{\prime}}\left(N^{\prime}\right)$ induces an isomorphism in homology. 
Proposition 14. Let $\mathcal{C}$ be an $\Sigma$-cofibrant $D G K$-module operad. The following functors preserve equivalences:

$$
\begin{gathered}
\widetilde{B}_{\mathcal{C}}(?): D G \text {-C-algebras } \rightarrow \mathcal{C} \text {-D-structures } \\
C_{\Delta}(?): \mathcal{C} \text {-D-structures } \rightarrow D G \text {-C } \text {-algebras } .
\end{gathered}
$$

There exist natural equivalences

$$
\begin{aligned}
& C_{\Delta}\left(\widetilde{B}_{\mathcal{C}}(?)\right) \rightarrow I d: D G \text {-C-algebras } \rightarrow \text { DG-C-algebras } \\
& \widetilde{B}_{\mathcal{C}}\left(C_{\Delta}(?)\right) \rightarrow I d: \mathcal{C} \text {-D-structures } \rightarrow \mathcal{C} \text {-D-structures }
\end{aligned}
$$

Proof. We have $C_{\Delta} \widetilde{B}_{\mathcal{C}}(A)=B_{\mathcal{C}}(A)$ by Proposition 9, so the natural equivalence of DG-C-algebras (13) is established by Proposition 8 , Since equivalences of $\mathcal{C}$-D-structures $f:(N, \Delta) \rightarrow\left(N^{\prime}, \Delta^{\prime}\right)$ are defined as equivalences of the corresponding $\mathrm{DG}-\mathcal{C}$-algebras, this also implies that (11) preserves equivalences.

The functor (12) on morphisms is defined by $f \mapsto \bar{f}$. It preserves equivalences by definition.

To construct the natural equivalence $\epsilon$ of (14), again, we need to construct $\bar{\epsilon}$, i.e. a natural equivalence of DG- $\mathcal{C}$-algebras

$$
B_{\mathcal{C}}\left(C_{\Delta}(?)\right) \rightarrow C_{\Delta}(?)
$$

which is the natural equivalence of Proposition 8 applied to $C_{\Delta}$.

\section{Derived CAtegories}

The material covered in this section is well known. We cover it for the lack of convenient reference.

Definition 15. Let $C$ be a category, and let $\mathcal{E} \subseteq \operatorname{Mor}(C)$ be an arbitrary class of morphism, which we call equivalences. A derived category $D C$ is a category together with a functor

$$
\Phi: C \rightarrow D C
$$

such that

$$
\phi \in \mathcal{E} \Rightarrow \Phi(\phi) \text { is an isomorphism, }
$$

and for every functor $F: C \rightarrow B$ such that

$$
\phi \in \mathcal{E} \Rightarrow F(\phi) \text { is an isomorphism, }
$$

there exists a functor $D F: D C \rightarrow D$ and a natural isomorphism

$$
\eta: F \stackrel{\cong}{\longrightarrow} D F \circ \Phi
$$


which is further unique in the following sense: For any other functor $D F^{\prime}: D C \rightarrow D$ and a natural isomorphism

$$
\eta^{\prime}: F \stackrel{\cong}{\longrightarrow} D F^{\prime} \circ \Phi
$$

there exists a unique natural isomorphism

$$
\xi: D F \stackrel{\cong}{\longrightarrow} D F^{\prime}
$$

such that

$$
\xi(\Phi)=\eta^{\prime} \circ \eta^{-1}
$$

Note that the existence of a derived category is not automatic, and in particular cannot be proved by the usual algebraic "localization" argument because $\mathcal{E}$ is only a class, not necessarily a set. In certain cases a derived category is known to exist, for example when $\mathcal{E}$ is the class of equivalences in a Quillen model structure [4].

Proposition 16. If a derived category exists then it is unique in the sense that for two functors $\Phi: C \rightarrow D C, \Phi^{\prime}: C \rightarrow D^{\prime} C$ both satisfying the condition of Definition 15, there exists a natural equivalence

$$
\begin{aligned}
& A: D C \rightarrow D^{\prime} C, B: D^{\prime} C \rightarrow D C, \\
& \epsilon: I d \stackrel{\cong}{\longrightarrow} B A, \quad \zeta: I d \stackrel{\cong}{\longrightarrow} A B
\end{aligned}
$$

and natural isomorphisms

$$
\eta: \Phi^{\prime} \stackrel{\cong}{\longrightarrow} A \Phi, \quad \kappa: \Phi \stackrel{\cong}{\longrightarrow} B \circ \Phi^{\prime}
$$

such that

$$
(B \eta) \circ \kappa=\epsilon \Phi,(A \kappa) \circ \eta=\zeta \Phi^{\prime} .
$$

Furthermore, these data are unique in the following sense: For any other choice of the data (16), (17) satisfying (18), (19) (which we will decorate with a (?) ${ }^{\circ}$ to make a distinction), there are unique isomorphisms

$$
\alpha: A \stackrel{\cong}{\longrightarrow} A^{\circ}, \quad \beta: B \stackrel{\cong}{\longrightarrow} B \circ B^{\circ}
$$

such that

$$
\begin{gathered}
I d=\left(\zeta^{\circ}\right)^{-1} \circ\left(\beta A^{\circ}\right) \circ(B \alpha) \circ \zeta, \\
I d=\left(\epsilon^{\circ}\right)^{-1} \circ\left(\alpha B^{\circ}\right) \circ(A \beta) \circ \epsilon, \\
\eta^{\circ}=\alpha \Phi \circ \eta, \kappa^{\circ}=\beta \Phi^{\prime} \circ \kappa .
\end{gathered}
$$


Proof. One obtains the transformations $\eta, \kappa$ as special cases of the $\eta$ in Definition [15, and the $\epsilon, \zeta, \alpha, \beta$ as special cases of the $\xi$ of Definition 15. The diagrams are then special cases of (15) and the uniqueness of $\xi$ in Definition 15.

Lemma 17. Suppose $C_{1}, C_{2}$ are categories with classes of equivalences $\mathcal{E}_{1}, \mathcal{E}_{2}$. Suppose that

(1) $\left(C_{1}, \mathcal{E}_{1}\right)$ has a derived category $\Phi_{1}: C_{1} \rightarrow D C_{1}$.

(2) There exists a pair of functors $F: C_{1} \rightarrow C_{2}, G: C_{2} \rightarrow C_{1}$ which preserve equivalences, and natural equivalences

$$
\epsilon: F G \stackrel{\sim}{\longrightarrow} I d, \quad \zeta: G F \stackrel{\sim}{\longrightarrow} I d .
$$

Then $C_{2}$ has a derived category $\Phi_{2}: C_{2} \rightarrow D C_{2}$, and there exist functors

$$
D F: D C_{1} \rightarrow D C_{2}, D G: D C_{2} \rightarrow D C_{1}
$$

and natural isomorphisms

$$
\begin{gathered}
D \epsilon: D F D G \stackrel{\cong}{\longrightarrow} I d, \quad D \zeta: D G D F \stackrel{\cong}{\longrightarrow} I d, \\
\kappa: \Phi_{2} F \stackrel{\cong}{\longrightarrow} D F \Phi, \quad \lambda: \Phi_{1} G \stackrel{\cong}{\longrightarrow} D G \Phi_{2}
\end{gathered}
$$

satisfying the identities

$$
\begin{aligned}
& I d=D \zeta \circ(D G \kappa) \circ(\lambda F) \circ \epsilon^{-1}, \\
& I d=D \epsilon \circ(D F \lambda) \circ(\kappa F) \circ \zeta^{-1} .
\end{aligned}
$$

Comment: We say that $F, G$ induce an equivalence of derived categories. There is also a uniqueness statement which we omit.

Proof. Put $D C_{2}:=D C_{1}, \Phi_{2}:=\Phi_{1} G$. We may then define $D F=$ $D G:=I d$, and $\lambda:=I d$. To define $\kappa: \Phi_{1} G F \rightarrow \Phi_{1}$, put $\kappa:=\Phi_{1} \zeta$. The natural isomorphisms $D \zeta, D \epsilon$ are then defined as special cases of the $\xi$ of Definition [15.

Comment: The fact that we defined $D C_{2}:=D C_{1}$ does not affect the force of this Lemma, since a derived category is only defined up to equivalence; the force of the statement is in the functors and natural isomorphisms involved.

The following is the main result of this section. 
Theorem 18. Let $\mathcal{C}$ be an $\Sigma$-cofibrant $D G K$-module operad. There exists a derived category of $\mathcal{C}$-D-structures, and the functors of Proposition 14 induce an equivalence of derived categories.

Proof. An immediate consequence of Proposition 14 and Lemma 17. the derived category of DG $\mathcal{C}$-algebras follows from the standard closed model structure (where cofibrations are retracts of relative cell objects).

\section{Appendix: The Multi-Sorted CAse}

In this apppendix, we will briefly explain what has to be changed in the definitions and theorems of this paper to cover the case of multisorted operads (equivalently, multicategories, cf. Elmendorf, Mandell [5]).

We are given a set $S$ (in the preceding sections, we assumed $S$ is a 1-element set). An $S$-sorted operad $\mathcal{C}$ over $K$-Chain is defined in the same way as an operad over $K$-Chain, with $(n)$ replaced by a system $\mathcal{C}(n)_{s_{1}, \ldots, s_{n} ; t}$ where $s_{1}, \ldots, s_{n}, t$ range independently over all elements of $S$. For every $s \in S$, there is a unit

$$
K \rightarrow \mathcal{C}(1)_{s ; s}
$$

The operations (1) become

$$
\begin{aligned}
& \mathcal{C}\left(n_{1}\right)_{s_{1,1}, \ldots, s_{1, n_{1}} ; t_{1}} \otimes \cdots \otimes \mathcal{C}\left(n_{k}\right)_{s_{k, 1}, \ldots, s_{k, n_{k}} ; t_{1}} \otimes \mathcal{C}(k)_{t_{1}, \ldots, t_{k} ; u} \\
& \rightarrow \mathcal{C}\left(n_{1}+\cdots+n_{k}\right)_{s_{1,1}, \ldots, s_{k, n_{k}} ; u} .
\end{aligned}
$$

Similarly,

$$
\gamma_{j}: \mathcal{C}(k)_{s_{1}, \ldots, s_{k} ; t_{j}} \otimes \mathcal{C}(n)_{t_{1}, \ldots, t_{n} ; u} \rightarrow \mathcal{C}(n+k-1)_{t_{1}, \ldots, t_{j-1}, s_{1}, \ldots, s_{k}, t_{j+1}, \ldots t_{n} ; u} .
$$

For an object $M$ of $(K \text {-Chain })^{S}$ (the $S$-fold product of categories), the $S$-sorted operad $\mathcal{H}_{M}$ is defined by

$$
\mathcal{H}_{M}(n)_{s_{1}, \ldots, s_{n} ; t}=\operatorname{Hom}\left(M_{s_{1}} \otimes \cdots \otimes M_{s_{n}} ; M_{t}\right)
$$

and for an $S$-sorted operad $\mathcal{C}$, a morphism of $S$-sorted operads (2) defines an $S$-sorted $D G$-C-algebra $M$.

Accordingly, the monad $C$ in $(K \text {-chain })^{S}$ corresponding to $\mathcal{C}$ is defined by

$$
(C X)_{t}=\bigoplus_{n \geq 0} \bigoplus_{s_{1}, \ldots, s_{n} \in S}\left(X_{s_{1}} \otimes \cdots \otimes X_{s_{n}}\right) \otimes_{\Sigma_{n}} \mathcal{C}(n)_{s_{1}, \ldots, s_{n} ; t} .
$$


$\Sigma$-cofibrancy on an $S$-sorted operad is defined to mean that

$$
\bigoplus_{\left(s_{1}, \ldots, s_{n}\right) \in \mathcal{O}} \mathcal{C}(n)_{s_{1}, \ldots, s_{n} ; t}
$$

be a $K\left[\Sigma_{n}\right]$-cell chain complex for every $t \in S$ and every $\Sigma_{n}$-orbit $\mathcal{O}$ of the product $S^{n}$.

Regarding trees, an $S$-sorted tree $(n, s, L, f)$ is a tree $(n, S, L)$ with a map

$$
f: \mathbf{n} \rightarrow S \text {. }
$$

An intertwining operation $\sigma: \mathbf{n} \rightarrow \mathbf{n}$ between $S$-sorted trees $(n, s, L, f)$ and $\left(n^{\prime}, s^{\prime}, L^{\prime}, f^{\prime}\right)$ must satisfy $f^{\prime} \sigma=f$. Similarly, $S$-sorted successor trees of $(n, s, L, f)$ are $\left(n_{i}, s_{i}, L_{i}, f_{i}\right)$ where

$$
f_{i}(j)=f\left(j+k_{i-1}\right) .
$$

All other definitions and statements regarding trees remain completely analogous for $S$-sorted trees.

Regarding the augmented Ginzburg-Kapranov bar construction, the definition (44) is modified by replacing

$$
\bigoplus
$$

by

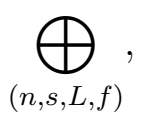

$\mathcal{C}(k)$ by $\mathcal{C}(k)_{f\left(i_{1}\right), \ldots, f\left(i_{k}\right) ; f(i)}$ where

$$
s\left(i_{1}\right)=\cdots=s\left(i_{k}\right)=i
$$

and $A$ by $A_{f(i)}$.

Thereby, $\widetilde{B}_{\mathcal{C}}(A)$ is an object of $(K-\text { Chain })^{S}\left(\widetilde{B}_{\mathcal{C}}(A)_{s}\right.$ is the summand of the modified (4) over $f(n)=s)$.

Propositions 4, 8 translate readily to $(K-\text { Chain })^{S}$. $B_{\mathcal{C}}(A)$ becomes an $S$-sorted DG-C-algebra.

To define an $S$-sorted $D$-structure (Definition 10), $\delta$ is an $S$-tuple of morphisms of graded $K$-modules, $\Delta$ is required to give $C N$ a structure of an $S$-sorted $\mathcal{C}$-algebra. In Definition [13, $f_{0}$ is an $S$-tuple of morphisms of graded $K$-modules, $\bar{f}$ is a morphism of $S$-sorted DG-Calgebras.

Again, in the spirit of [9], the category of $S$-sorted $\mathcal{C}$ - $D$-structures is equivalent to the full subcategory of the category of $\mathcal{C}$-algebras which are free $C$-algebras after forgetting differentials.

Definition 13, Proposition 14 and Theorem 18 remain precisely analogous, replacing DG-C-algebras by $S$-sorted DG-C-algebras. 


\section{REFERENCES}

[1] M.Anel, A.Joyal: Sweedler Theoy for (co)algebras and the bar-cobar constructions, arXiv:1309.6952

[2] J.M.Boardman, R.M.Vogt: Homotopy invariant algebraic structures on topological spaces, Lecture Notes in Mathematics, Vol. 347. Springer-Verlag, BerlinNew York, 1973

[3] W.G.Dwyer, D.M.Kan: Homotopy theory and simplicial groupoids, Nederl. Akad. Wetensch. Indag. Math., 46, (1984), 379 - 385

[4] W.Dwyer, J. Spalinski: Homotopy theories and model categories, Handbook of algebraic topology, 73126, North-Holland, Amsterdam, 1995

[5] A.D.Elmendorf, M.l A. Mandell: Rings, modules, and algebras in infinite loop space theory, Advances in Math. 205,1 (2006), 163-228

[6] B.Fresse: Operadic cobar constructions, cylinder objects and homotopy morphisms of algebras over operads, Alpine perspectives on algebraic topology, Contemp. Math. 504, AMS, Providence, 2009, pp. 125-188

[7] W.Gan: Koszul Duality for Dioperads, Math. Res. Letters 10 (2003) 109-124

[8] E.Getzler, J.D.S.Jones: $A_{\infty}$-algebras and the cyclic bar complex, Illinois J. Math. 34 (1990), no. 2, 256-283

[9] E.Getzler, J.D.S.Jones: Operads, homotopy algebra and iterated integrals for double loop spaces, preprint, 1994

[10] V.Ginzburg, M.Kapranov: Koszul duality for operads, Duke Math. J. 76 (1994) 203-272

[11] J.Hirsh, J.Milles: Curved Koszul duality theory, Math. Ann. 354 (2012), 14651520

[12] P.Hu: Higher string topology on general spaces, Proc. London Math. Soc. (3) 93 (2006), no. 2, 515-544

[13] P.Hu, I.Kriz, A.A.Voronov: On Kontsevich's Hochschild cohomology conjecture. (English summary) Compos. Math. 142 (2006), no. 1, 143-168

[14] M.Kontsevich: Deformations of algebras over operads and the Deligne conjecture, Conférence Moshé Flato 1999, Vol. I (Dijon), 255-307, Math. Phys. Stud., 21, Kluwer Acad. Publ., Dordrecht, 2000

[15] M.Kontsevich, Y.Soibelman: Deformations of algebras over operads and the Deligne conjecture, Conférence Moshé Flato 1999, Vol. I (Dijon), 255-307, Math. Phys. Stud., 21, Kluwer Acad. Publ., Dordrecht, 2000

[16] I.Kriz, J.P.May:Operads, algebras, modules and motives, Astrisque No. 233 (1995)

[17] R.Lipshitz, P.S.Ozsváth, D.P.Thurston: Bimodules in bordered Heegaard Floer homology, arXiv: 1003.0598

[18] J.-L. Loday, B. Vallette: Algebraic Operads, Grundlehren der mathematischen Wissenschaften, Springer Verlag, 2012

[19] J.P. May: The geometry of iterated loop spaces, Lectures Notes in Mathematics, Vol. 271. Springer-Verlag, Berlin-New York, 1972

[20] J.P.May, R.Thomason: The uniqueness of infinite loop space machines, Topology 17 (1978), no. 3, 205224

[21] P.Ozsváth, Z.Szabó: Holomorphic disks and topological invariants for closed three-manifolds, Ann. of Math. (2) 159 (2004), no. 3, 10271158

[22] A.Polishchuk, L.Positelski: Quadratic algebras, Univ. Lecture Series 37, AMS, Providence, 2005 
[23] S. Priddy: Koszul resolutions, Trans. Amer. Math. Soc. 1521970 39-60

[24] D. Quillen: On the (co)homology of commutative rings, Homotopy theories and model categories. Handbook of algebraic topology, 73126, North-Holland, Amsterdam, 1995. 1970 Applications of Categorical Algebra (Proc. Sympos. Pure Math., Vol. XVII, New York, 1968) pp. 6587. Amer. Math. Soc., Providence, R.I.

[25] B.Vallette: A Koszul duality for PROPs, Trans. Amer. Math.Soc. 152 (1970) $39-60$ 